\title{
Strategy Abandonment Effects in Cued Recall
}

\author{
Stephanie A. Robinson*a, Amy A. Overman ${ }^{a,}$, \& Joseph D.W. Stephens ${ }^{b}$ \\ ${ }^{a}$ Department of Psychology, Elon University, NC \\ ${ }^{b}$ Department of Psychology, North Carolina A\&T State University, NC \\ Student:srobins1@brandeis.edu \\ Mentor:aoverman@elon.edu
}

\begin{abstract}
Decades of research have investigated the effects of encoding strategies in the formation of associations in memory. Despite this, it is not known whether or how changes in the use of strategies within a brief time span may affect memory. For example, what is the effect on memory of abandoning a recent strategy or switching to a different strategy? The present study systematically varied the strategies used by participants in two closely-spaced associative memory tasks. Results indicated that intentional abandonment of a verbal (sentence-generation) strategy had disproportionately negative consequences on memory for semantically unrelated word pairs. The findings suggest that memory encoding is affected by differences in strategy use across recent memory tasks, and have implications for effective use of memory strategies in practical settings.
\end{abstract}

\section{KEYWORDS}

Cued Recall; Encoding Strategies; Inhibition

\section{INTRODUCTION}

The intentional use of appropriate encoding strategies to improve memory for associations between items has been welldocumented $^{1}$. Much of the initial work on memory encoding strategies compared the relative effectiveness of specific strategies, such as mental imagery and sentence formation ${ }^{2,3}$. Other work has focused on examining factors that influence individual differences in strategy selection and use $e^{4,5,6,7}$, as well as the effects of strategy training, often in special populations ${ }^{8,9,10,11}$.

The existing literature has provided important insights into the effectiveness of memory encoding strategies within the context of individual memory tasks. However, memory tasks in everyday life often do not exist in isolation. For example, one may need to perform more than one memorization task within a short time frame, such as studying for an exam or orienting to a new work environment. There is currently little research that has investigated strategy use across more than one closely-spaced memory task. Thus, it is not known whether abandoning an encoding strategy, switching between encoding strategies, or practicing use of an encoding strategy confers any benefits or costs across associative memory tasks.

Successive memory tasks with the same or different strategies may be approached as a type of task-switching, in which the particular encoding strategy instructions constitute the participant's task. Therefore, the task-switching literature provides a framework for understanding the ways in which cognitive processing at a given moment is influenced by recent processing ${ }^{12}$. For example, performance on a current task can be impaired by inhibition of a prior task with similar processing demands ${ }^{13}$. These findings in the task-switching literature are relevant to the question of whether a current memory encoding task is influenced by an encoding task that preceded it.

With regard to switching of memory strategies or abandonment of a memory strategy, the previously-used strategy must be inhibited during the use of the current strategy. The inhibition of prior encoding strategies could impair current encoding if some of the same cognitive processes are contributing to both tasks. For example, if an explicitly verbal encoding strategy is being inhibited, the inhibition of the strategy may also inhibit verbal encoding processes that are not specific to that strategy. This could result in performance that is worse than if the prior strategy had not been employed at all (and therefore does not need to be inhibited). In addition to the possible direct inhibition of relevant encoding processes, the attentional and cognitive control resources involved in the inhibition may reduce the general processing resources available for encoding.

There may also be immediate effects of repeating the use of a particular strategy. For example, when memorizing two sets of associates within a single study session, it might be beneficial to use the same strategy for both, to take advantage of practice effects. For example, extensive training on a memory strategy has been shown to improve use of the strategy across multiple sessions ${ }^{8}$. On the other hand, immediate strategy repetition within an experimental session might lead to detrimental effects such as interference or cognitive fatigue ${ }^{14}$. If associative memory benefits from using a strategy due to familiarity with an encoding 
technique, then the benefit would be expected to increase with repeated use (practice/training) of the encoding strategy. Thus, it remains unclear how memory performance is affected by strategy practice and strategy switching. This study aimed to compare the effects, if any, of strategy addition or abandonment, strategy switching, and strategy repetition on cued recall.

\section{METHODS AND PROCEDURES}

\section{Participants}

One hundred eighty-nine participants were recruited from university psychology courses (mean age $=19.12$ years; range $=18$-23 years; 155 females, 34 males). They either received course credit or were entered into a lottery to win a gift card to a local store. All participants were native English speakers and reported no history of neurological or psychiatric disorders. Prior to the experiment, all participants provided written informed consent to participate using consent forms approved by the Institutional Review Board of the university. After study participation was complete, all participants were given a verbal and written debriefing.

\section{Materials}

The experimental stimuli consisted of 128 words selected from the University of South Florida word association database ${ }^{15}$. The words were selected to produce a heterogeneous assortment in terms of length, word frequency, part of speech, concreteness, and imageability, with the constraint that half of the words were selected to produce semantically related pairs according to the association norms (mean relatedness $=.77$; range $=.65-.94$ ), and the other half were selected to ensure no semantic relationship (mean relatedness $=0.00$; range $=0.00$ ). The pairs were separated into two study lists for use in the cued-recall tasks. The lists were counterbalanced so that each pair was equally likely to be used in the first or second study-test phase for each participant, in order to prevent any systematic variation in the individual word properties across conditions ${ }^{16}$.

\section{Design}

Three encoding strategies were selected for this experiment because of prior research that has established that the three basic categories of self-initiated strategies are visual, semantic, and shallow ${ }^{4}$. The use of a visual word-learning task allowed us to employ each of these strategies: visual, semantic, no strategy. In order to examine any effects of changing between encoding strategies, we used each possible pairing of three encoding strategy instructions: imagery, sentence, and no strategy, resulting in a 9 (encoding strategy order; between-subjects) X 2 (semantic relatedness; within-subjects) mixed model design.

For all conditions, care was taken to thoroughly discuss with the participant what was expected. Instructions were first presented on the computer screen for the participant to read. Then, participants were asked to explain the task back to the experimenter and the experimenter emphasized task expectations and corrected any errors in understanding. If there were errors, participants were asked again to explain the instructions to the experimenter. Then, prior to using a prescribed strategy, participants completed a practice trial of the task. After the practice trial they described what they were doing during the practice trials to the experimenter. If participants were not correctly employing a strategy after the first practice trial or reported using a strategy when they were instructed not to do so, the instructions were re-explained and discussed with the participant and then the participant attempted a second practice trial. On the second practice trial, no participants reported using an incorrect strategy or using a strategy in the no strategy task. Participants were randomly assigned to one of nine possible strategy sequences resulting from the combination of the three sets of strategy instructions across two encoding phases (See Table 1). There were an average of 21 participants per condition, although the exact number in each condition varied slightly due to random assignment.

\section{Second List}

\begin{tabular}{lccc}
\cline { 2 - 4 } First List & No Strategy & Sentence & Image \\
\hline No Strategy & Repetition $(\mathrm{n}=19)$ & Addition $(\mathrm{n}=21)$ & Addition $(\mathrm{n}=22)$ \\
Sentence & Abandonment $(\mathrm{n}=21)$ & Repetition $(\mathrm{n}=22)$ & Switching $(\mathrm{n}=20)$ \\
Image & Abandonment $(\mathrm{n}=21)$ & Switching $(\mathrm{n}=20)$ & Repetition $(\mathrm{n}=23)$ \\
\hline
\end{tabular}

Table 1. Strategy Sequences Across Study Lists

Note. Each participant was given one of nine possible sequences of encoding strategy instructions across two cuedrecall tasks. Effects of strategy addition, strategy abandonment, strategy switching, and strategy repetition were analysed in terms of the performance differences observed between the two cued-recall tasks in each of the nine sequences.

For the imagery encoding strategy, participants were asked to visualize the words in a scene: for example, the pair SALT-PEPPER (semantically related) might be visualized as salt and pepper shakers on a table; the pair TOUCAN-FORK (semantically unrelated) might be visualized as a toucan using a fork to eat. For the sentence formation strategy, participants were asked to think of a 
sentence using both words in the word pair. Using the same word pairs above, for the semantically-related pair, the participant could form the sentence "Could you please put the salt and pepper on the dinner table?", and for the semantically-unrelated pair, the participant could form the sentence "The toucan stole my fork during our picnic". For no strategy, participants were instructed not to use any strategy and examples were given of strategies that should not be used (e.g., sentence formation, imagery, rote repetition).

\section{Procedure}

The experiment was conducted using E-Prime software (Psychology Software Tools, Sharpsburg, PA). The stimuli were presented side-by-side in black Courier New 16-point font on a white background. Participants were seated at a computer and told that they would be seeing a list of word pairs and would later be asked to remember what they saw. Each study list contained 32 pairs of words. Sixteen related pairs and 16 unrelated pairs were randomly assigned to each study list. Half were pairs of words that were semantically related and half were pairs of words that were arbitrarily assigned. Pairs were presented for seven seconds. At the end of the list, participants completed a three-minute simple addition task before proceeding to the cued recall task. For the cued recall task, one of the words from each pair in the list was presented on a sheet of paper and the participant was instructed to fill in the corresponding word in the pair. All the pairs from the study list were tested. Thus, there were 16 cue words from related pairs and 16 cue words from unrelated pairs presented on the cued recall test. After participating in the first studytest phase, the participants waited for two minutes and then proceeded to the second study-test phase, which followed the same format: written and verbal instructions, study, distractor task, and then test.

\section{RESULTS}

Of particular interest for the current study was whether the use of a strategy (or no strategy) on a second cued recall test would be influenced by the use of a strategy (or no strategy) on a prior cued recall test. Thus, the focus was not on the relative efficacies of the encoding strategies but rather on the differences in performance observed with changes in strategy use across successive tasks. We considered three possibilities for such influences: 1) whether performance changed when the same strategy was used twice (strategy repetition); 2) whether the difference between a strategy and no strategy depended on whether the no-strategy task came first (strategy addition) versus second (strategy abandonment); and 3) whether the difference between the two strategies depended on the order of the two strategies (strategy switching). Each of these possibilities was examined by analysing the groups of participants relevant to each comparison. Because each group of participants completed two tests, we particularly focused the analyses on the change in performance from one test to another, thereby controlling for variations in overall performance across individual participants and groups.

\section{Strategy repetition}

For the question of whether the effect of any strategy changed with repetition, we compared the three groups that had the same strategy (or lack thereof) twice - i.e., No Strategy (N-N group), Sentence (S-S group), and Imagery (I-I group). We analysed performance in a 2 (test) X 2 (relatedness) X 3 (strategy) ANOVA. When the same strategy was used twice, there was no change in performance between the first and second tests, $F(1,61)=1.74, M S E=.028, p=.19$. There was also no difference between strategy types, $F(2,61)=.934, M S E=.098, p=.40$, and the were no interactions (all $p>.25$ ). There was a main effect of relatedness, $F(1,61)=238.6, M S E=6.18, p<.001, \eta_{\mathrm{p}}{ }^{2}=.80$, such that cued recall was better for target words that were semantically related to their cues (see below for an overall analysis of the effect of relatedness across all strategy sequences). Thus, performance did not significantly change with strategy repetition.

\section{Strategy addition versus abandonment}

For the question of whether the effect of a strategy versus no strategy depended on whether the no-strategy task occurred first or second, we compared the group that had No Strategy in the first study list, followed by Imagery in the second study list (i.e., the N-I group), with the group that had Imagery in the first study list, then No Strategy in the second study list (i.e., the I-N group). We also compared the group that had No Strategy in the first study list, followed by Sentence in the second study list (i.e., the N-S group), with the group that had Sentence in the first study list, followed by No Strategy in the second study list (i.e., the S-N group).

The comparison between the N-I and I-N groups is displayed in Figure 1. The difference in performance between the two cuedrecall tests was computed for each participant as $\mathrm{p}$ (Correct|Imagery) $-\mathrm{p}$ (Correct $\mid$ No Strategy). Thus, the data represent the relative benefit of Imagery across the two strategy orders. These data were analysed in a 2 (relatedness) X 2 (order) ANOVA with relatedness as a within-subjects factor and order as a between-subjects factor. The effect of relatedness was significant, $F(1,41)=$ 20.6, MSE $=.394, p<.001, \eta_{\mathrm{p}}^{2}=.34$, indicating that the Imagery strategy benefitted recall of target words that were semantically unrelated to their cue words more than it benefitted cued recall of semantically-related targets. There was no significant effect of order, $F(1,41)=1.15, M S E=.063, p=.29$, and no significant interaction, $F(1,41)=3.05$, MSE $=.058, p=.09$, suggesting that the benefit to recall performance caused by adding the Imagery strategy was similar to the harm caused by abandoning it. 


\section{Imagery vs. No Strategy}

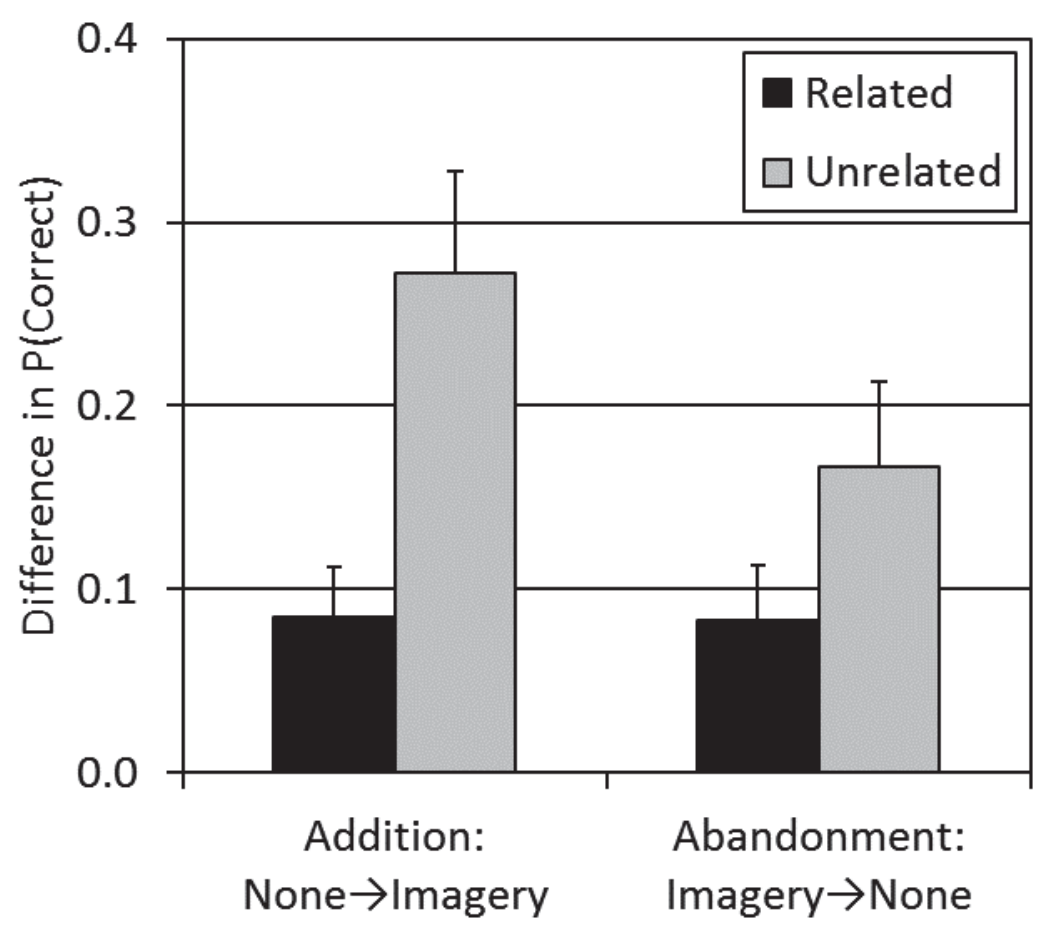

Figure 1. Cued-recall performance with imagery versus no strategy, across strategy addition and abandonment conditions. Difference scores were computed as proportion correct with imagery minus proportion correct with no strategy, for each participant. Error bars represent standard error of the mean.

The comparison between the N-S and S-N groups is displayed in Figure 2. As with N-I and I-N, the difference in performance between strategy and no strategy was computed as the relative benefit of Sentence, $\mathrm{p}($ Correct $\mid$ Sentence $)-\mathrm{p}($ Correct $\mid$ No Strategy). The data were analysed in a 2 (relatedness) X 2 (order) ANOVA with relatedness as a within-subjects factor and order as a between-subjects factor. The main effect of relatedness was not significant, $F(1,40)=2.03, M S E=.086, p=.16$, and there was no significant effect of order, $F(1,40)=.03$, MSE $=.002, p=.87$. However, there was a significant interaction of relatedness $\mathrm{X}$ order, $F(1,40)=11.2, M S E=.474, p=.002, \eta_{\mathrm{p}}^{2}=.22$. Inspection of the data in Figure 2 indicates that the addition of the Sentence strategy mainly caused a benefit in cued recall of target words that were semantically related to their cue words, whereas abandonment of the Sentence strategy mainly caused a cost to the cued recall of semantically-unrelated words. These observations were examined in detail using one-sample $t$-tests comparing the means in each condition to zero. For the N-S group, the change in performance was significantly different than zero for related words, $t(20)=3.85, p=.001, d=.84$, and unrelated words, $t(20)=$ $2.09, p=.049, d=.46$. For the S-N group, the change in performance between strategies was significant only for the unrelated words, $t(20)=4.33, p<.001, d=.94$, and not for related words, $t(20)=.36, p=.73$. 


\section{Sentence vs. No Strategy}

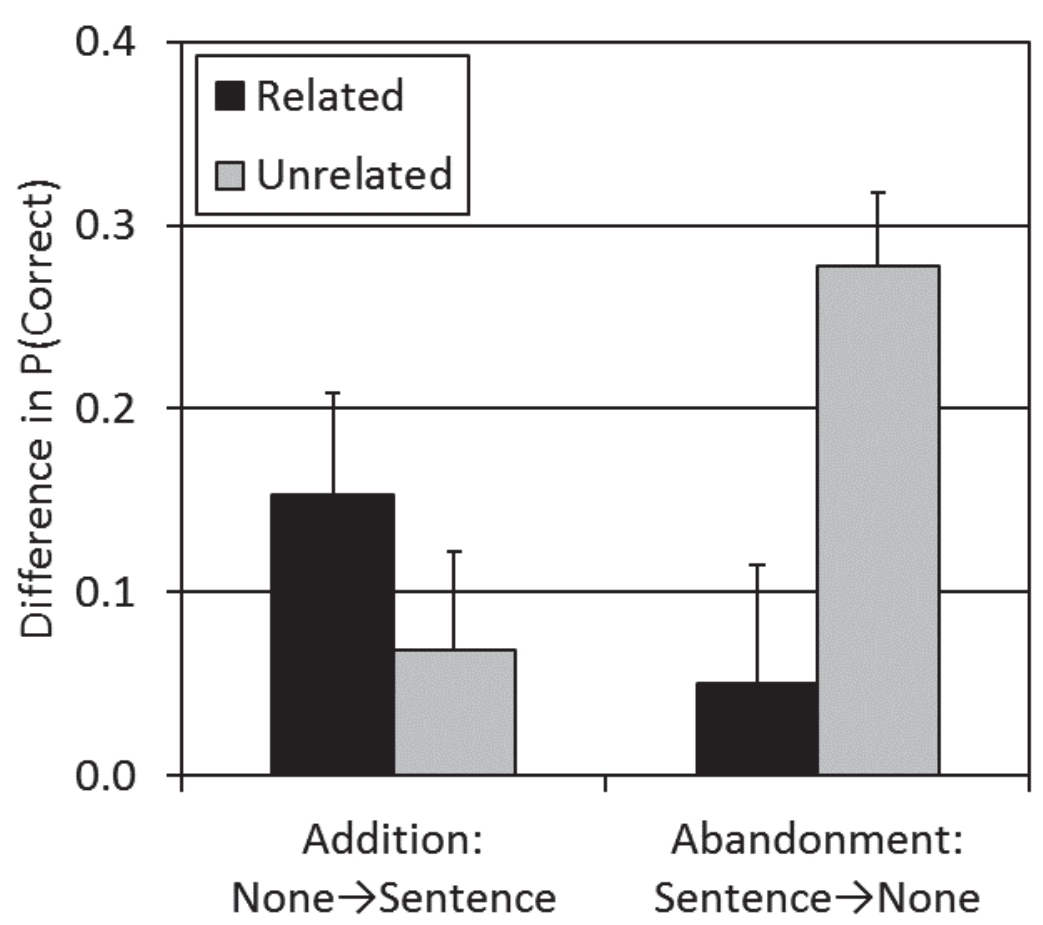

Figure 2. Cued-recall performance with sentence generation versus no strategy, across strategy addition and abandonment conditions. Difference scores were computed as proportion correct with sentence generation minus proportion correct with no strategy, for each participant. Error bars represent standard error of the mean.

\section{Strategy switching}

For the question of whether the difference in performance between the two strategies depended on strategy order, we compared the group that had the Sentence strategy first, followed by Imagery (S-I group), with the group that had Imagery first, then Sentence (I-S group). Similar to the analyses of strategy addition versus abandonment, we examined the change in performance between strategies as the benefit of Imagery over Sentence, $\mathrm{p}$ (Correct $\mid$ Imagery) $-\mathrm{p}$ (Correct| Sentence). We then analysed these data in a 2 (relatedness) X 2 (order) ANOVA. There was no main effect of relatedness, $F(1,38)=3.48, M S E=.127, p=.07$, and there was no main effect of order, $F(1,38)=.07, M S E=.002, p=.79$. There was also no interaction of relatedness $\mathrm{X}$ order, $F(1,38)=.03, M S E=.001, p=.86$. Thus, the order in which the two strategies were used did not affect cued-recall performance.

\section{Overall effect of relatedness}

In addition to the questions of interest analysed above, the results were analysed with respect to the overall effect of relatedness. Across both tests, target words that were semantically related to their cue words were recalled more often than those that were unrelated. A 2 (test list) X 2 (relatedness) ANOVA on number of words correctly recalled found a significant effect of relatedness, $F(1,188)=704.3, M S E=18.2, p<.001, \eta_{\mathrm{p}}^{2}=.79$, no effect of list, $F(1,188)=.573, M S E=.019, p=.45$, and no interaction, $F(1,188)=1.56, M S E=.032, p=.24$. This effect of relatedness is in line with prior studies (e.g., Jenkins \& Russell, 1952).

\section{Overall effects of strategy type}

Although the main questions of interest for the current study had to do with differences in performance between tests according to strategy use, we also compared the strategies to each other overall, for comparison to prior work on study strategies. Because different participants received different strategy sequences, the overall effectiveness of each strategy was examined separately within each test list. Thus, strategy was analysed as a between-subjects factor in two ANOVAs, one for each test list. For the first test list, the effect of strategy was significant, $F(2,186)=14.8, M S E=.380, p<.001, \eta_{\mathrm{p}}^{2}=.14$. Pairwise Bonferroni-corrected post-hoc comparisons indicated that Imagery was better than no strategy, $p<.001$, Sentence was better than no strategy, $p<.001$, but Imagery and Sentence did not differ, $p=1.0$. For the second test list, the effect of strategy was significant, $F(2,186)=11.4$, 
$M S E=.294, p<.001, \eta_{\mathrm{p}}^{2}=.11$. Pairwise Bonferroni-corrected post-hoc comparisons indicated that Imagery was better than no strategy, $p<.001$, and Imagery was better than Sentence, $p=.037$. Due to the correction for multiple comparisons, sentence was not significantly better than no strategy, $p=.076$. The overall greater effectiveness of Imagery was in line with the well-established literature on memory strategies ${ }^{2,3}$. The fact that the statistical differences between strategies were not equivalent between the two tests may be attributable to the order effects of N-S versus S-N described above.

\section{DISCUSSION \& CONCLUSIONS}

The purpose of the current study was to examine the effects, if any, of changes in memory strategy use across two cued-recall tasks performed within a single experimental session. Little prior research has investigated how the immediate addition, abandonment, switching, or repetition of memory strategies affects the usefulness of those strategies in boosting memory performance.

Interestingly, we found that the effect of abandoning the sentence-formation strategy did not mirror the effect of adding the sentence-formation strategy. Unrelated words were disproportionately affected by abandonment of the sentence strategy, in comparison to how they were affected by addition of the sentence strategy. This finding was in contrast to the results for the mental imagery strategy. Abandonment of the imagery strategy on the second task had nearly the exact opposite effect as adding the strategy on the second task after using no strategy on the first.

Although it may seem counterintuitive that abandonment of a sentence formation strategy would have different effects than abandonment of an imagery strategy, the results may be interpreted in light of the relationship between the task and the strategy. The memory tasks in this study were verbal in nature (i.e., encoding of word pairs). Participants who used a sentence strategy on the first task, and were then instructed to stop using the strategy, likely did so by inhibiting some of their verbal processing during the second task. Thus, participants who abandoned the sentence strategy had fewer verbal processing resources available for encoding word pairs during the subsequent no-strategy task. In contrast, participants who used an imagery strategy on the first task, and then were instructed to stop using the strategy, did not need to inhibit any verbal processing because the first strategy they used did not require any verbal processing.

It may be argued that we do not know exactly what participants were doing in the no-strategy task, and thus strategy abandonment might be considered another form of strategy switching, in which participants simply switched to idiosyncratic strategies of their own choosing. Nonetheless, the important result remains that this particular form of switching led to a different result in the case of Sentence to No Strategy switching than in the other forms of switching considered here. Based on this finding, we argue that the instruction to abandon the sentence strategy, without providing an alternative strategy such as imagery, leads to a subsequent inhibition of verbal processing in whatever idiosyncratic encoding processes are subsequently employed in the next task. An additional possibility to consider is whether encoding strategy instructions might also affect retrieval processing during the subsequent cued recall task. For example, participants who are instructed to abandon a sentence strategy might also inhibit verbal processes used in recollection of the target word at test. Participants who are abandoning an imagery strategy might not inhibit such retrieval processes as they seem unrelated to the strategy being abandoned.

Other than the effects of strategy abandonment, our study indicated that the effect of a strategy on the second cued-recall task was independent of what strategy was used on the previous cued-recall task. Immediate repetition did not lead to significant improvements, and the effect of changing strategies did not depend on the order in which the strategies were used. It is important to note that, in the conditions that involved immediate repetition of a strategy, we were specifically interested in possible practice effects, rather than in training effects. Thus, participants were not instructed or encouraged to become more proficient at the use of the strategy as in some prior studies ${ }^{8}$.

This study is an important first step toward understanding how the abandonment of prior strategies may lead to inhibitory effects on subsequent strategies, which result in a detriment to memory performance. These results also have implications for real-world scenarios in which people engage in more than one study task within a brief period of time, such as studying for examinations. Further research should build on this study both in terms of examining the cognitive mechanisms involved in strategy abandonment as well as how to optimize strategy use in learning contexts.

\section{ACKNOWLEDGMENTS}

Joseph Stephens was supported by Air Force Research Laboratory and OSD under agreement number FA8750-15-2-0116. The U.S. Government is authorized to reproduce and distribute reprints for Governmental purposes notwithstanding any copyright notation thereon. The views and conclusions contained herein are those of the authors and should not be interpreted as necessarily representing the official policies or endorsements, either expressed or implied, of Air Force Research Laboratory and OSD or the U.S. Government. 


\section{REFERENCES}

1. Richardson, J. T. E. (1998). The availability and effectiveness of reported mediators in associative learning: A historical review and an experimental investigation. Psychonomic Bulletin \& Review, 5, 597-614.

2. Paivio, A. (1969). Mental imagery in associative learning and memory. Psychological Review, 76, 241-263.

3. Bower, G. H., \& Winzenz, D. (1970). Comparison of associative learning strategies. Psychonomic Science, 20, 119-120.

4. Dunlosky, J., \& Hertzog, C. (1998). Aging and deficits in associative memory: what is the role of strategy production? Psychology \& Aging, 13, 597-607.

5. Dunlosky, J., \& Hertzog, C. (2001). Measuring strategy production during associative learning: The relative utility of concurrent versus retrospective reports. Memory \& Cognition, 29, 247-253.

6. Kirchhoff, B. A. (2009). Individual differences in episodic memory: The role of self-initiated encoding strategies. The Neuroscientist, 15, 166-179.

7. Kuhlmann, B. G., \& Touron, D. R. (2012). Mediator-based encoding strategies in source monitoring in young and older adults. Journal of Experimental Psychology: Learning, Memory, and Cognition, 38, 1352.

8. Brehmer, Y., Shing, Y. L., Heekeren, H. R., Lindenberger, U., \& Bäckman, L. (2016). Training-induced changes in subsequent-memory effects: No major differences among children, younger adults, and older adults. NeuroImage, 131, 214-225.

9. Hampstead, B. M., Stringer, A. Y., Stilla, R. F., Giddens, M., \& Sathian, K. (2012). Mnemonic strategy training partially restores hippocampal activity in patients with mild cognitive impairment. Hippocampus, 22, 1652-1658.

10. Patrick, J., Morgan, P. L., Smy, V., Tiley, L., Seeby, H., Patrick, T., \& Evans, J. (2015). The influence of training and experience on memory strategy. Memory \& Cognition, 43, 775-787.

11. Rebok, G. W., Carlson, M. C., \& Langbaum, J. B. (2007). Training and maintaining memory abilities in healthy older adults: traditional and novel approaches. The Journals of Gerontology Series B: Psychological Sciences and Social Sciences, 62, $53-61$.

12. Kiesel, A., Steinhauser, M., Wendt, M., Falkenstein, M., Jost, K., Philipp, A. M., \& Koch, I. (2010). Control and interference in task switching-A review. Psychological Bulletin, 136, 849-874.

13. Philipp, A. M., \& Koch, I. (2006). Task inhibition and task repetition in task switching. European Journal of Cognitive Psychology, 18, 624-639.

14. Van der Linden, D., Frese, M., \& Meijman, T. F. (2003). Mental fatigue and the control of cognitive processes: Effects on perseveration and planning. Acta Psychologica, 113, 45-65.

15. Nelson, D. L., McEvoy, C. L., \& Schreiber, T. A. (2004). The University of South Florida free association, rhyme, and word fragment norms. Behavior Research Methods, Instruments, \& Computers, 36, 402-407.

16. Madan, C., Glaholt, M., \& Caplan, J. (2010). The influence of item properties on association-memory. Journal of Memory and Language, 63, 46-63.

17. Jenkins, J. J., \& Russell, W. A. (1952). Associative clustering during recall. Journal of Abnormal and Social Psychology, 47, 818821.

\section{ABOUT THE STUDENT AUTHOR}

Stephanie Robinson graduated from Elon University with a B.A. in Psychology. Stephanie completed a Master's in Psychology at the Catholic University of America where she continued to explore memory and learning strategies. She is currently completing a Ph.D. in Psychology at Brandeis University in Waltham, MA with a focus on behavioural and psychosocial differences in cognition across the lifespan and investigating intervention methods to ameliorate age-related cognitive decline.

\section{PRESS SUMMARY}

For decades, researchers have investigated strategies to facilitate memory; however, it not yet known if continuous use of the same strategy is more useful than using a novel strategy. That is, if an individual was studying for two exams, would it be helpful to use the same strategies to memorize the material (strategy repetition; i.e., practice), or is it more helpful to use a new strategy for new material (strategy abandonment)? This study demonstrated that the effects of switching strategies was dependent qualified by the relatedness of the material. This study provides an important first step to understanding how switching study strategies could negatively impact memory. 